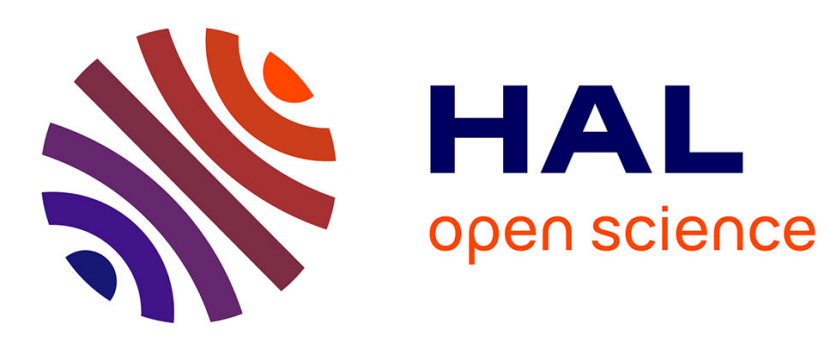

\title{
Smooth Visual-Coverage Path Planning for Escort Missions using UAVs
}

Kaouther Moussa, Hilton Tnunay, Ahmad Hably, Nicolas Marchand

\section{To cite this version:}

Kaouther Moussa, Hilton Tnunay, Ahmad Hably, Nicolas Marchand. Smooth Visual-Coverage Path Planning for Escort Missions using UAVs. IECON 2021 - The 47th Annual Conference of the IEEE Industrial Electronics Society, Oct 2021, Toronto (virtual ), Canada. 10.1109/IECON48115.2021.9589197 . hal-03359203

\section{HAL Id: hal-03359203 https://hal.science/hal-03359203}

Submitted on 30 Sep 2021

HAL is a multi-disciplinary open access archive for the deposit and dissemination of scientific research documents, whether they are published or not. The documents may come from teaching and research institutions in France or abroad, or from public or private research centers.
L'archive ouverte pluridisciplinaire HAL, est destinée au dépôt et à la diffusion de documents scientifiques de niveau recherche, publiés ou non, émanant des établissements d'enseignement et de recherche français ou étrangers, des laboratoires publics ou privés. 


\title{
Smooth Visual-Coverage Path Planning for Escort Missions using UAVs
}

\author{
Kaouther Moussa ${ }^{1}$, Hilton Tnunay ${ }^{2}$, Ahmad Hably $^{2}$, Nicolas Marchand ${ }^{2}$ \\ ${ }^{1}$ Univ. Polytechnique Hauts-de-France, LAMIH, CNRS, UMR 8201, F-59313 Valenciennes, France \\ kaouther.moussa@uphf.fr \\ ${ }^{2}$ Univ. Grenoble Alpes, Grenoble INP, GIPSA-Lab, CNRS, France \\ $\{$ ishak.tnunay, ahmad.hably, nicolas.marchand\} @ gipsa-lab.fr
}

\begin{abstract}
This paper investigates the trajectory generation problem for a fleet of Unmanned Aerial Vehicles (UAVs) performing escort missions with respect to a convoy with a timevarying velocity. Using camera-equipped UAVs, we propose a framework allowing to adapt the UAVs altitudes to achieve a trade-off between the size of the covered surface and the image resolution by solving an optimization problem. Furthermore, we perform polynomial refinement based on the generated planar way-points to yield a minimum-jerk time-parameterized trajectory with dynamical constraints on the virtual leader to follow. The numerical simulations presented in this paper show the effectiveness of the optimization algorithm in adapting the UAVs altitudes to satisfy a compromise between the size of the covered area and its corresponding image resolution.

Index Terms-Escort missions, Path planning, Optimization, Surface coverage, Image resolution
\end{abstract}

\section{INTRODUCTION}

In the last decades, multi-robot systems have witnessed widespread interest in the research community. The applications related to those systems are diverse, for example, security, search and rescue, environmental monitoring, and agriculture [1]. The use of multiple robots might have several advantages, for example, increasing the flexibility and efficiency of the mission execution as well as enhancing the mission performance with respect to a single robot system.

According to [2], consistent research has been devoted to applications such as exploration and mapping of unknown environments, but only a few works addressed the escorting problem explicitly. The latter consists of surrounding a moving target by reducing its escape windows or similarly protecting a target by reducing the intrusion of any external agent. The escorting problem has different applications, for example, surveillance and security systems, military robotics or entertainment robotics.

In this paper, we are interested in designing a mission manager for convoy escorting problems. The UAVs are equipped with cameras with a given resolution. The escorting mission is such that the UAV fleet tracks the convoy trajectory using a virtual leader topology while performing surface coverage by means of cameras in order to detect any external intruder and to guarantee convoy security. The size of the surface to be covered depends on the speed of the convoy in order to

This work was supported by the TAMOS (TActical Multi-Objective Swarming UAVs) project. guarantee a sufficient visibility distance allowing the convoy to anticipate an action with respect to an intruder.

Coverage Path Planning (CPP) consists in designing robots paths in order to explore a structure or an environment and to gather information. It has been developed for performing different real-world inspection applications in both aerial and mobile robotics, for example, structural health monitoring, robot manipulation, autonomous lawn mowing, floor cleaning, and stereo reconstruction [3], [4], [5], [6], [7]. These applications usually require the robot to completely cover a bounded region using either cameras or sensors, which help to significantly improve the flexibility and the efficiency of the coverage system.

According to [3], the majority of existing CPP approaches focus on minimizing the path distance while performing surface coverage, whereas only a few studies incorporate the quality of images in the path optimization problem even though inspection missions commonly require high-quality images. The importance of collecting high-quality images is the ability to perform image analysis and extract reliable information, especially in the case of surveillance missions, where security is of high importance.

In the last years, the development of automatic visual inspection and surveillance algorithms has drawn attention since manual inspection might be time-consuming and prone to human errors [8]. Some works in the literature focus on image processing algorithms in order to achieve visual inspection, see for example [8], [9] and [10]. However, in this paper, we focus on the path planning problem, and especially on optimizing the altitude of the UAVs, in order to achieve a trade-off between image quality and the size of the covered surface. Therefore, we do not investigate the processing of the collected images.

The escorting problem that we investigate in this paper is similar to visual CPP in the sense that the UAVs positions (in particular altitudes) are adapted in order to satisfy a given image resolution specification. However, it is important to highlight the fact that in escorting missions, the objective is not to generate a trajectory (for example, a zigzag trajectory shape) to cover an area. This is because the convoy movement imposes the UAVs planar trajectory ( $\mathrm{x}$ and $\mathrm{y}$ positions).

The common objective of escorting missions in the literature is to circulate around the boundary of a given target, using 
multiple robots, in order to create a virtual fence allowing to avoid internal or external agents crossing through the delimited area [11]. This problem has been dealt with from a control point of view in the literature, for example, in [12], [13] and [14], many cooperative control approaches has been applied. Furthermore, in [11] a distributed planning method with dynamics boundaries has been presented. However, these methods focus on task assignment and formation control problems and do not take into consideration the coverage quality using specific metrics such as the covered area size and the image resolution.

In this paper, we focus on the path planning problem for a fleet of UAV's during escort missions, using a virtual leader topology. The specificity of our work is to ensure dynamical coverage in the sense that we adapt the surface to be covered based on the convoy speed. Furthermore, we solve an optimization problem allowing to adapt the UAVs altitudes to the convoy speed to achieve a trade-off between the size of the covered surface and the quality of the images. The planar way-points of the UAVs are based either on the convoy movement map known as a priori or its estimated position and orientation, in the case where the convoy moves away from the planned path. Finally, based on these planar waypoints, we perform a polynomial refinement in order to derive polynomial trajectories minimizing the jerk and thereby the energy consumption while considering dynamical constraints related to the UAVs.

The main contribution of this paper is the integration of the optimization problem that allows to achieve a trade-off between image quality and the size of the covered area, providing thereby a framework for escort missions path planning with image resolution guarantees.

The paper is organized as follows, Section II presents the escorting mission problem and the camera settings. In Section III, we present the solution layout corresponding to the proposed framework. Section IV presents the simulation results with a particular focus on the optimization problem that we propose to solve. Finally, Section V summarizes the work presented in this paper and suggests some future perspectives.

\section{Problem Setup}

\section{A. Escorting missions: problem formulation}

As explained previously, the concept of escorting missions using multi-robot systems can have different interpretations, depending on the type of the target (threat or protection) and also the behavior of the formation (static or dynamic with respect to the convoy movement).

Let's consider a rectangular shape convoy with $N_{c}$ vehicles moving along a planned path with a given velocity. We have a fleet of $N$ UAVs that are equipped with cameras with a resolution of $1920 \times 1080$ pixels, distributed in a line formation. As illustrated in Fig. 1, we define the reference surface to be covered $\mathcal{S}_{0}$ as the sum of the two following surfaces:

1) $\mathcal{S}_{\text {Convoy }}$ : the surface around the convoy, it is defined by its geometry.

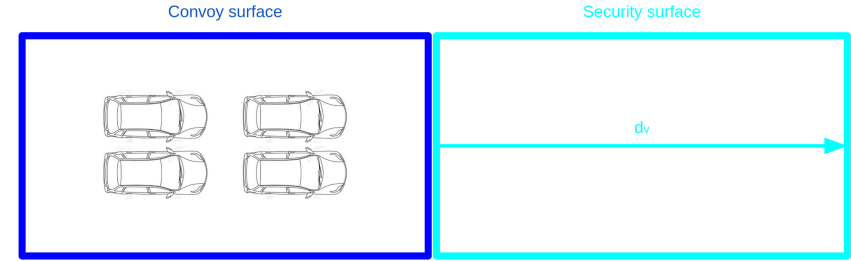

Fig. 1: Illustration of the escorting mission

2) $\mathcal{S}_{\text {Security }}$ : the surface to be covered ahead of the convoy for security reasons. This surface is defined based on the visibility distance $d_{v}$ depending on the convoy speed and will be presented subsequently.

$$
\mathcal{S}_{0}=\mathcal{S}_{\text {Convoy }}+\mathcal{S}_{\text {Security }}
$$

In this paper, the mission of escorting a convoy can be seen as the requirement of tracking the convoy trajectory while covering the reference surface by means of cameras and proper distribution of the UAVs. The convoy moves along a path that is known a priori; however, it might deviate from it, in which case a prediction of the trajectory might be performed.

Managing such convoy escorting missions involves the two following objectives:

1) Maximising the covered surface.

2) Maximising the image quality.

These two objectives are antagonistic, since maximising the surface to be covered means that we need to increase the UAVs altitudes, which increases the pixel size in the images deteriorating thereby the image quality. Inversely, maximising the image quality implies reducing the UAVs altitudes in order to decrease the pixel size, which reduces the size of the covered area.

This paper aims to design a mission manager that provides a time-parametrised trajectory of the formation and desired distances among the agents to execute the escorting operation while satisfying a trade-off between both antagonistic objectives. The trajectory and desired distance are then provided to the UAV's guidance control for execution.

\section{B. Camera settings}

Fig. 2 illustrates the field of view (FoV) of a camera having as angles $\alpha \times \beta$, the covered area being rectangular.
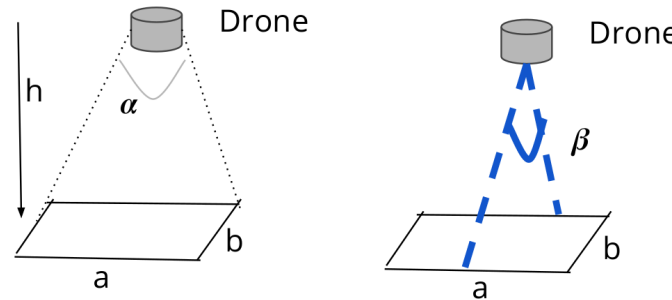

Fig. 2: Camera angles illustration

Given the camera angles $\alpha$ and $\beta$, we can compute the width and the length of the covered area as follows: 


$$
\begin{aligned}
& a=2 h \tan \left(\frac{\alpha}{2}\right) m \\
& b=2 h \tan \left(\frac{\beta}{2}\right) m
\end{aligned}
$$

where $h$ is the UAV altitude. The camera resolution is $1920 \times 1080$, therefore the pixel size of the covered area is the following:

$$
p_{s}=\frac{2000 a}{1920}
$$

\section{TRAJECTORY GENERATION FOR ESCORTING MISSIONS}

The problem of convoy escorting presented in this paper gathers two main objectives; the first one is tracking the convoy trajectory using a fleet of UAVs, whereas the second one is the surveillance (surface coverage) of this convoy using the cameras installed on the UAVs. And the second one consists of tracking them. Therefore, the solution that we propose in this case gathers both coverage planning and trajectory prediction. Fig. 3 illustrates the framework that we propose for managing escorting missions. This framework is composed of 4 main blocks:

1) Trajectory prediction: allows to generate the trajectory way-points of the 2D virtual leader ( $\mathrm{x}$ and $\mathrm{y}$ axes), either by following the specified map or by performing predictions

2) Polynomial generation: allows to generate a timeparametrized trajectory of the virtual leader, by minimizing the jerk and considering dynamical constraints

3) Surveillance optimization: allows to adapt the reference surface to be covered according to the convoy speed. Furthermore, it provides the UAVs altitude by solving the optimization problem describing the trade-off between surface coverage and image resolution.

4) Formation topology: allows to position the UAVs with respect to the virtual leader trajectory, by providing the distances between the agents and the virtual leader trajectory in the 2D plan ( $\mathrm{x}$ and $\mathrm{y}$ axes), these distances are called "Deltas" and denoted by $\delta_{x}^{i}$ and $\delta_{y}^{i}$ where $i=1, \ldots, N$, with $N$ being the number of UAVs

The framework that we propose adopts the virtual leader topology, by splitting the problem of determining the UAVs positions into two main problems. The first one consists in determining the $2 \mathrm{D}$ virtual leader positions that we denote $p_{x}^{l}$ and $p_{y}^{l}$, corresponding to the $x$ and $y$ axes respectively. The second problem consists in determining the UAVs altitude that achieves the trade-off between image quality and surface coverage.

\section{A. Trajectory prediction}

Let's denote the three-dimensional position and the heading of the UAVs by $p_{i}=\left[\begin{array}{lll}p_{x}^{i} & p_{y}^{i} & p_{z}^{i}\end{array}\right]^{\top}$ and $\psi_{i}$, respectively, for $i=1, \ldots, N$. We denote by $p_{c}=\left(x_{c}, y_{c}\right)^{\top}$ the $2 \mathrm{D}$ way-points of the convoy planned trajectory, and by $\hat{p}_{c}=\left(\hat{x}_{c}, \hat{y}_{c}\right)^{\top}$ the estimated position of the convoy. Furthermore, we denote by $\hat{v}_{c}$ and $\hat{\theta}$ the estimated speed and orientation of the convoy. We



Fig. 3: Overall scheme of the proposed algorithm

assume that $\hat{p}_{c}, \hat{v}_{c}, \hat{\theta}$ are provided by some embedded sensors. In order to simplify the problem, we assume that all the convoy vehicles have the same speed and orientation, approximating thereby the convoy by a single vehicle with a given speed and orientation.

The primary role of the virtual leader is to follow the same path as the convoy. Therefore, the virtual leader 2D way-points $\left(p_{x}^{l}, p_{y}^{l}\right)$ are determined according to the following algorithm, which allows to choose between the planned path $p_{c}$ or a prediction, based on a minimal tolerance distance $d_{t o l}$ between the planed and the estimated convoy position $p_{c}$ and $\hat{p}_{c}$, respectively.

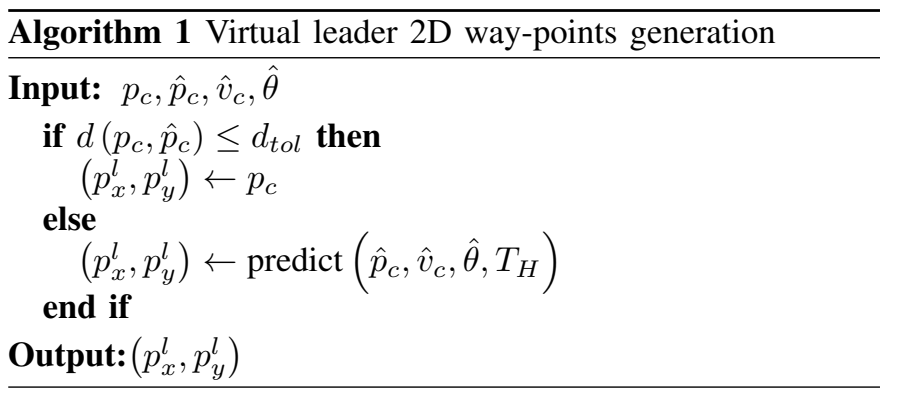

Where $d(\cdot, \cdot)$ is the euclidean distance between two points. The prediction is performed for a time horizon $T_{H}$ in a receding horizon way, by approximating the convoy movement by the following dynamics, with $\hat{p}_{c}$ as initial condition:

$$
\begin{aligned}
& \dot{x}_{c}=\hat{v}_{c} \cos \hat{\theta} \\
& \dot{y}_{c}=\hat{v}_{c} \sin \hat{\theta}
\end{aligned}
$$

Fig.4 shows the 2D trajectory corresponding to these profiles as well as the predicted trajectory; we can notice that they overlap. The next step is to generate a polynomial trajectory based on the 2D way-point provided by this block.

\section{B. Polynomial trajectories generation}

In this mission, a trajectory refinement process is executed by minimizing the performance index of the path derivatives 


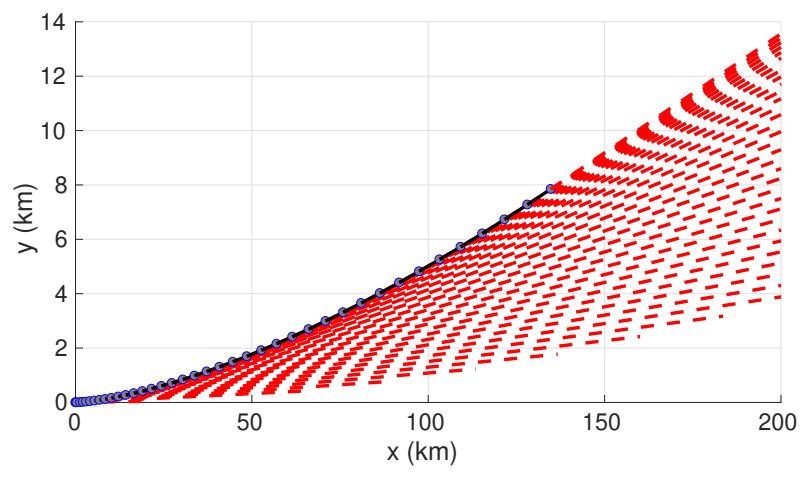

Fig. 4: The way-points of the convoy trajectory (grey circles), the predicted trajectories at time sample (dashed red lines) and the final predicted trajectory for the virtual leader (solid black)

to obtain a smooth time-parameterized trajectory that accommodates the dynamics limitation of the UAVs. Following the existing works in [15], [16] and [17] and [18], a constrained quadratic programming $(\mathrm{QP})$ problem is exploited to formulate and generate a minimum-jerk polynomial trajectory of the virtual leader.

Consider a set of $m$ time intervals connecting $m+1$ waypoints. The time-parametrised trajectory along these waypoints can be expressed as a piecewise polynomial function of order $k_{n}$ over $m$ intervals, $\sigma(t)=\left[p_{x}^{d}, p_{y}^{d}, p_{z}^{d}, \psi_{f}^{d}\right]^{\top}$, where

$$
\sigma(t)= \begin{cases}\sum_{j=0}^{k_{n}} x_{j 1} t^{j} & \text { for } t_{0} \leq t<t_{1} \\ \sum_{j=0}^{k_{n}} x_{j 2} t^{j} & \text { for } t_{2} \leq t<t_{2} \\ \cdots & \\ \sum_{j=0}^{k_{n}} x_{j m} t^{j} & \text { for } t_{m-1} \leq t \leq t_{m} .\end{cases}
$$

The polynomial coefficients of this function can be reparametrised into a decision vector $\hat{x} \in \mathbb{R}^{4 n m+1}$ with $\hat{x}=$ $\left[x_{1}, x_{2}, \ldots, x_{m}\right]$. The corresponding performance index for the jerk minimization of the trajectory is given by

$$
J=\int_{0}^{\tau} \gamma_{p}\left\|\frac{d^{3} \sigma(t)}{d t^{3}}\right\| d t=x^{\top} H x
$$

with $H$ denoting a Hessian matrix of to the desired penalty on the polynomial derivatives.

In this mission, we aim to accommodate the way-point, continuity and dynamics constraints. The first constraint ensures that the trajectory always reach the specified way-points, including the start and finish points. The start and finish points can be formulated via

$$
\begin{aligned}
A_{0}^{\text {start }} x_{0} & =b_{0}, \\
A_{\tau}^{\text {end }} x_{\tau} & =b_{\tau},
\end{aligned}
$$

and the remaining way-points via

$$
A_{i}^{\text {start }} x_{i}=b_{i}
$$

where $b_{i}$ is the $i$-th way-point to achieved by the virtual leader. The second mission corresponds to the continuity of the trajectories. This is to say that the start point of the $i+1$ segment aligns with the end of $i$-th segment. This constraint can be expressed by the following equality constraint:

$$
A_{i}^{\text {end }} x_{i}=A_{i+1}^{\text {start }} x_{i+1} .
$$

Matrices $A_{i}^{\text {start }}$ and $A_{i}^{\text {end }}$ are transformation matrices of the coefficient $x_{i}$ to the derivatives of the polynomial at the start and end points, respectively. The last constraints deals with the dynamics limitation of the UAVs to ensure that the refined trajectory is still dynamically feasible. The inequality to accommodate this constraint is formulated via

$$
A^{\mathrm{dyn}} \hat{x} \leq b^{\mathrm{dyn}},
$$

with $A^{\mathrm{dyn}}$ and $b^{\mathrm{dyn}}$ being the mapping matrix of the dynamics constraint and the vector containing the dynamics limitation of the leader, respectively. The maximum velocity and acceleration of the leader are imposed in our mission.

In order to solve the optimization problem, the computation method in [16] is employed due to its stability and computational efficiency. This trajectory refinement process yields a time-parameterized trajectory $\sigma$ of the virtual leader as given in (5), including its first derivative as the velocity and the second derivatives as the acceleration.

\section{Surveillance quality metrics}

The coverage quality criterion $J$ includes two metrics, the first one is the sum of the surfaces covered by all the UAVs:

$$
\mathcal{S}=\Sigma_{i=1}^{N} \mathcal{S}_{i}=\Sigma_{i=1}^{N} a_{i} b_{i}=\Sigma_{i=1}^{N} 4 h_{i}^{2} \tan \left(\frac{\alpha}{2}\right) \tan \left(\frac{\beta}{2}\right)
$$

Whereas the second one is the pixel size in the covered area:

$$
p_{s}=\frac{2000 h}{1920} \tan \left(\frac{\alpha}{2}\right)
$$

\section{Surveillance optimization}

Let's assume that all the UAVs are positioned at the same height $h$, the first metric can be simplified as follows:

$$
\mathcal{S}=4 N h^{2} \tan \left(\frac{\alpha}{2}\right) \tan \left(\frac{\beta}{2}\right)
$$

Furthermore, we consider the following constraint:

$$
h_{\min }<h<h_{\max }
$$

where $h_{\min }$ is the altitude minimal bound, allowing to make the UAV fly above the convoy and to consider the constraint on the minimal surface to cover per UAV. $h_{\max }$ is the altitude maximal bound, allowing to ensure the security of the UAVs in the flying space, and also to consider the minimal resolution constraint.

The reference surface $\mathcal{S}_{0}$ to be covered has been defined previously as the sum of $\mathcal{S}_{\text {Convoy }}$ and $\mathcal{S}_{\text {Security. }}$ The security surface $\mathcal{S}_{\text {Security }}$ depends on the vehicle speed and the nature of the line, and is defined based on the visibility distance [19], see Table I.

The convoy surface $\mathcal{S}_{\text {Convoy }}$ being constant, the evolution $\mathcal{S}_{0}$ depends only on $\mathcal{S}_{\text {Security }}$. Fig. 5 shows the evolution of $\mathcal{S}_{0}$ in function of the convoy speed $v_{c}$. 


\begin{tabular}{|c|c|c|c|c|c|c|c|}
\hline$v_{c}(\mathrm{~km} / \mathrm{h})$ & 30 & 50 & 60 & 70 & 80 & 90 & 100 \\
\hline$d_{v}$ straight line $(\mathrm{m})$ & 25 & 50 & 65 & 85 & 105 & 130 & 160 \\
\hline$d_{v}$ curved line $(\mathrm{m})$ & 26.5 & 55 & 72 & 95 & 121 & 151 & 187 \\
\hline
\end{tabular}

TABLE I: Visibility distance $d_{v}$ in function of the convoy speed $v_{c}$



Fig. 5: Evolution of $\mathcal{S}_{0}$ in function of the convoy speed $v_{c}$

As explained previously, the two objectives of surface coverage and image resolution are antagonistic. Therefore, we need to find the UAVs altitude $h$ which achieves a trade-off between surface coverage and a high image resolution. Let's define the following cost function with $\rho_{1}, \rho_{2}>0$ :

$$
\begin{aligned}
J(h)= & \rho_{1}\left(\mathcal{S}-\mathcal{S}_{0}\right)^{2}+\rho_{2} p_{s} \\
= & \rho_{1}\left(4 N h^{2} \tan \left(\frac{\alpha}{2}\right) \tan \left(\frac{\beta}{2}\right)-\mathcal{S}_{0}\right)^{2}+ \\
& \rho_{2}\left(\frac{2000 h}{1920} \tan \left(\frac{\alpha}{2}\right)\right)
\end{aligned}
$$

This cost contains two penalty terms allowing to express both objective of maximizing the surface and the image resolution. The first one is achieved by minimizing the difference between the covered surface $\mathcal{S}$ and the reference surface $\mathcal{S}_{0}$, while the second one is achieved by minimizing the pixel size. Therefore, in order to achieve the trade-off, we need to minimize the previous cost for given weights $\rho_{1}$ and $\rho_{2}$ under the following constraint:

$$
h_{\min }<h<h_{\max }
$$

\section{E. Formation topology}

In this mission, we exploit the virtual leader based UAV formation topology. Virtual-leader based topology has successfully been applied to robot formation, for example, as reported in [20].

Consider a network graph $\mathcal{G}_{f}=\left(\mathcal{V}_{f}, \mathcal{E}_{f}\right)$ where the vertices $\mathcal{V}_{f}=\{0,1, \ldots, N\}$ contains all the agents, both the virtual and physical; and the edges $\mathcal{E}_{f} \subseteq \mathcal{V}_{f} \times \mathcal{V}_{f}$ represents their connectivity. The formation leader is also referred to as the virtual agent indexed $i=0$, while followers are the physical UAVs indexed $i=1,2, \ldots, N$. Employing the virtual-leader based formation allows us to design a trajectory for the virtual leader and the desired distance among the agents - we no longer need to generate the trajectory of all UAVs. The illustration of this virtual leader-based topology can be found in Fig. 6.

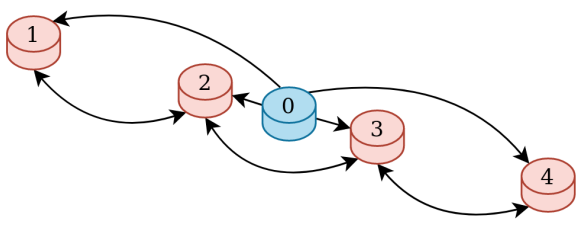

Fig. 6: Graph connectivity of a virtual leader (blue) and four followers (red)

In the escort mission requiring the UAVs to move in a formation to cover a moving area, the geometric formation shape is designed such that there is no gap among the area of the image coverage to improve the surveillance efficiency. Since the camera angles are non-adjustable, the image quality can only be controlled by adjusting the UAV altitude. However, the altitude changing also requires adjustment on the planar $x$ and $y$ positions to prevent any coverage gap. If a highresolution image is requested, then the UAVs need to fly at a low altitude, requiring them to fly close to each other. If a lower-resolution image is requested, then the UAVs may fly at a higher altitude, and the distance among them can be sufficiently large as long as there is no coverage gap. The trade-off in this performance has been discussed in the previous sections.

\section{Simulation Results}

The penalty term $\rho_{2}$ in the cost represents the importance of considering the image resolution. Therefore, we define three levels of importance for the resolution of the images, low, medium and high, corresponding to 3 different numerical values of $\rho_{2}$. In the following figures, the iterations correspond to different time samples and thereby to different convoy speeds. The optimization problem runs continuously in order to adapt the altitude for each convoy speed, defining a given reference surface. We can see in Fig.7 that the UAVs altitude increases as the image quality importance decreases. Furthermore, we can notice in Fig.8 that as the image quality importance decreases, the covered surface gets closer to the reference surface, deteriorating thereby the image quality, as we can see in Fig.9, the pixel size increasing meaning that the image quality is reduced.

\section{Conclusion}

This paper has presented a strategy for an autonomous surveillance system consisting of a fleet of camera-equipped UAVs to perform an escort mission with time-varying velocity. The proposed technique has exploited the image geometry to compute the distance between the UAVs and their altitude. An optimization problem has been formulated and solved to determine the optimal value that satisfies the trade-off between the size of the covered surface and the image resolution. Furthermore, the presented algorithm has also utilised a trajectory 


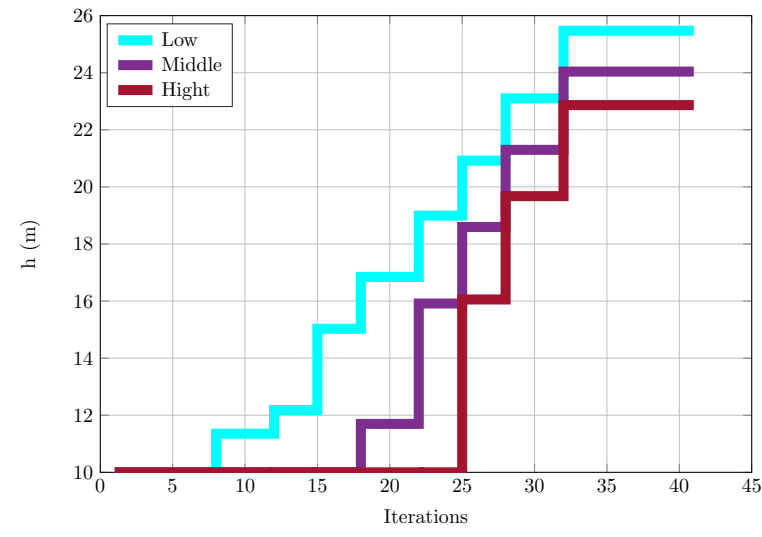

Fig. 7: Evolution of the UAVs altitude

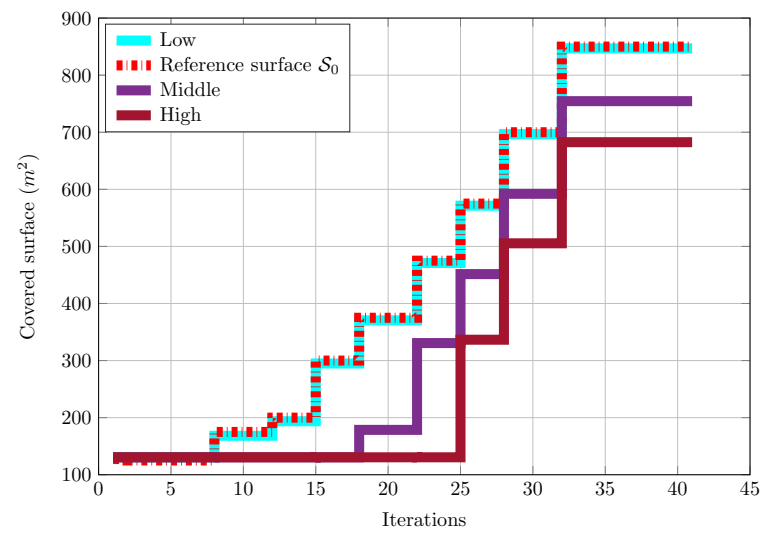

Fig. 8: Evolution of the covered surface

optimization problem that accommodates UAV's dynamical constraints to calculate a minimum-jerk trajectory to be followed by the fleet. Finally, a set of numerical simulations have validated the effectiveness of the algorithm.

Since we assumed in this paper that the number of UAVs is either chosen by the user or evaluated by a supervisor module, a future perspective for this work would be to include the number of UAVs as an unknown variable in the optimization

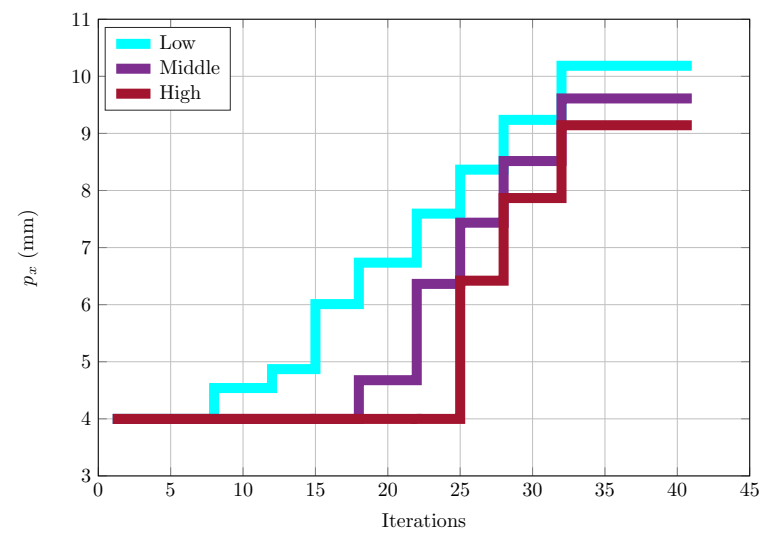

Fig. 9: Evolution of the pixel size problem in order to determine the optimal number with respect to the mission metrics.

\section{REFERENCES}

[1] K. Garapati, J. J. Roldán, M. Garzón, J. del Cerro, A. Barrientos, A game of drones: Game theoretic approaches for multi-robot task allocation in security missions, in: A. Ollero, A. Sanfeliu, L. Montano, N. Lau, C. Cardeira (Eds.), ROBOT 2017: Third Iberian Robotics Conference, Springer International Publishing, Cham, 2018, pp. 855-866.

[2] G. Antonelli, F. Arrichiello, S. Chiaverini, The entrapment/escorting mission, IEEE Robotics Automation Magazine 15 (1) (2008) 22-29.

[3] Z. Shang, J. Bradley, Z. Shen, A co-optimal coverage path planning method for aerial scanning of complex structures, Expert Systems with Applications 158 (2020) 113535.

[4] T.-K. Lee, S.-H. Baek, Y.-H. Choi, S.-Y. Oh, Smooth coverage path planning and control of mobile robots based on high-resolution grid map representation, Robotics and Autonomous Systems 59 (10) (2011) $801-812$.

[5] R. Funada, M. Santos, T. Gencho, J. Yamauchi, M. Fujita, M. Egerstedt, Visual Coverage Maintenance for Quadcopters Using Nonsmooth Barrier Functions, Proceedings - IEEE International Conference on Robotics and Automation (2020) 3255-3261.

[6] E. Galceran, M. Carreras, A survey on coverage path planning for robotics, Robotics and Autonomous Systems 61 (12) (2013) 1258-1276.

[7] S. S. Mansouri, C. Kanellakis, E. Fresk, D. Kominiak, G. Nikolakopoulos, Cooperative coverage path planning for visual inspection, Control Engineering Practice 74 (2018) 118-131.

[8] A hybrid vision-based surface coverage measurement method for robotic inspection, Robotics and Computer-Integrated Manufacturing 57 (2019) $138-145$.

[9] L. C. Vieira, R. de Almeida, Application of computer vision methods to estimate the coverage of peen formed plates 43 (2) (2010) 743-749.

[10] L. Shahid, F. Janabi-Sharifi, P. Keenan, Image segmentation techniques for real-time coverage measurement in shot peening processes, International Journal of Advanced Manufacturing Technology 91 (1-4) (2017) 859-867.

[11] A. Jahn, R. J. Alitappeh, D. Saldaña, L. C. A. Pimenta, A. G. Santos, M. F. M. Campos, Distributed multi-robot coordination for dynamic perimeter surveillance in uncertain environments, in: 2017 IEEE International Conference on Robotics and Automation (ICRA), 2017, pp. 273-278.

[12] S. Gao, R. Song, Y. Li, Cooperative control of multiple nonholonomic robots for escorting and patrolling mission based on vector field, IEEE Access 6 (2018) 41883-41891.

[13] J. Zhang, J. Sha, G. Han, J. Liu, Y. Qian, A cooperative-controlbased underwater target escorting mechanism with multiple autonomous underwater vehicles for underwater internet of things, IEEE Internet of Things Journal 8 (6) (2021) 4403-4416.

[14] L. Ma, Cooperative target tracking by altering uavs' linear and angular velocities, in: 2019 International Conference on Unmanned Aircraft Systems (ICUAS), 2019, pp. 69-78.

[15] D. Mellinger, V. Kumar, Minimum snap trajectory generation and control for quadrotors, Proceedings - IEEE International Conference on Robotics and Automation (2011) 2520-2525.

[16] C. Richter, B. Adam, R. Nicholas, Polynomial Trajectory Planning for Aggressive Quadrotor Flight in Dense Indoor Environments, Cailiao Yanjiu Xuebao/Chinese Journal of Materials Research 17 (5) (2013) $459-465$.

[17] M. W. Mueller, M. Hehn, R. Dandrea, A Computationally Efficient Motion Primitive for Quadrocopter Trajectory Generation, IEEE Transactions on Robotics 31 (6) (2015) 1294-1310.

[18] A. Bry, C. Richter, A. Bachrach, N. Roy, Aggressive Flight of FixedWing and Quadrotor Aircraft in Dense Indoor Environments 37 (June) (2015) 969-1002.

[19] http://catalogue.setra.fr/documents/Cataloguesetra/0005/Dtrf-0005572/ DT5572.pdf.

[20] F. Bullo, J. Cortés, S. Martínez, Distributed control of robotic networks: A mathematical approach to motion coordination algorithms, 2009. 